\title{
Construction of the pion scalar form factor from few poles and zero
}

\author{
Robert Kamiński ${ }^{1, \star}$, Stanislav Dubnicka ${ }^{2}, Z_{\text {Zuzana Dubnickova }}^{3}$, and Andrej Liptaj ${ }^{2}$ \\ ${ }^{1}$ Institute of Nuclear Physics PAS, Kraków, Poland \\ ${ }^{2}$ Institute of Physics SAS, Bratislava, Slovak Republic \\ ${ }^{3}$ Department of Theoretical Physics, Comenius University, Bratislava, Slovak Republic
}

\begin{abstract}
Construction and analysis of the pion scalar-isoscalar form factor in the elastic region is presented. Precise $S$-wave $\pi \pi$ scattering phase shifts generated by dispersive analysis of experimental data with imposed crossing symmetry condition are used. Final result for values of the $f_{0}(500)$ meson mass and width, $m_{\sigma}=(487 \pm 31) \mathrm{MeV} ; \Gamma_{\sigma}=$ $(542 \pm 60) \mathrm{MeV}$ is compatible with the results from dispersive analyses of the Bern and Madrid-Kraków groups to be considered now as the most reliable values of the $f_{0}(500)$ scalar meson parameters. Parameters of the $f_{0}(980)$, although lying almost on the $K \bar{K}$ threshold also agree with values predicted by these two groups.
\end{abstract}

\section{Introduction}

From the first identification of lightest hadronic resonance with vacuum quantum numbers $0^{++}$i.e. $f_{0}(500)$ in 1974 , a lot of work has been done and many papers to be concerned of this scalar meson are published up to now. However, only recently a clarification of this controversial situation with $f_{0}(500)$ has been achieved in the dispersive analyses done by the Bern $[1,2]$ and by the MadridKraków group [3, 4], which are now considered to be the most reliable determinations of the $f_{0}(500)$ scalar meson parameters.

In the paper [5] another method for a determination of the $f_{0}(500)$ scalar meson parameters by means of the pion scalar-isoscalar form factor analysis in the elastic region has been proposed. The parameters were, however, fitted to the inaccurate, and in some region even contradicting experimental data on the S-wave isoscalar $\pi \pi$-scattering phase shift. So, the obtained mass and width of the $f_{0}(500)$ scalar meson can be considered only to demonstrate the existence of $f_{0}(500)$, however to be far away from the true values of the mass and width of this meson. The same concerns meson $f_{0}(980)$ whose parameters have been found to be very far from indicated by Particle Data Group [6].

Here, in the presented analysis, an ultimate analysis of the pion scalar form factor in the elastic region is proposed with true $S$-wave isoscalar $\pi \pi$ scattering phase shift data with theoretical errors, to be generated by the GKPY Roy-like equations derived and presented in [3].

Final result for values of the $f_{0}(500)$ meson mass and width, $m_{\sigma}=(487 \pm 31) \mathrm{MeV} ; \Gamma_{\sigma}=(542 \pm 60)$ $\mathrm{MeV}$ is recovered to be consistent with Bern [2] and the Madrid-Kraków group [4], though determined in a completely different way.

\footnotetext{
^e-mail: robert.kaminski@ifj.edu.pl
} 


\section{Methodology}

The pion scalar-isoscalar form factor $(\mathrm{FF}) \Gamma_{\pi}(t)$ is defined by the parameterization of the matrix element of the scalar quark density

$$
<\pi^{i}\left(p_{2}\right)|\widehat{m}(\bar{u} u+\bar{d} d)| \pi^{j}\left(p_{1}\right)>=\delta^{i j} \Gamma_{\pi}(t) \text { where } t=\left(p_{2}-p_{1}\right)^{2} \text { and } \widehat{m}=\frac{1}{2}\left(m_{u}+m_{d}\right) .
$$

It posses all known properties of the pion vector electromagnetic FF $F_{\pi}(t)$ like analyticity in $t$ plane (beside cuts on the positive real axis from two-pion threshold $t=4 m_{\pi}^{2}$ to $+\infty$ ), elastic unitarity, asymptotic behavior $\Gamma_{\pi}(t)_{|t| \rightarrow \infty} \sim \frac{1}{t}$, reality condition $\Gamma_{\pi}^{*}(t)=\Gamma_{\pi}\left(t^{*}\right)$ and normalization to the pion sigma term value $\Gamma(0)=(0.99 \pm 0.02) m_{\pi}^{2}$ to be predicted by the $\chi P T$ [7].

The analyticity of the pion scalar FF in $t$-plane and asymptotic behavior (through the Cauchy formula) allow to derive dispersion relation with one subtraction

$$
\Gamma_{\pi}(t)=P_{n}(t) \exp \left[\frac{t}{\pi} \int_{4 m_{\pi}^{2}}^{\infty} \frac{\delta_{0}^{0}\left(t^{\prime}\right)}{t^{\prime}\left(t^{\prime}-t\right)} d t^{\prime}\right] .
$$

In the elastic region ( $\sqrt{t}<K \bar{K}$ threshold) one can express $\Gamma_{\pi}(t)$ in the form of a rational function

$$
\Gamma_{\pi}(q)=\frac{\sum_{n=0}^{M} a_{n} q^{n}}{\prod_{i=1}^{N}\left(q-q_{i}\right)},
$$

which leads to the parameterization

$$
\tan \delta_{\Gamma}(t)=\frac{A_{1} q+A_{3} q^{3}+A_{5} q^{5}+A_{7} q^{7}+\ldots}{1+A_{2} q^{2}+A_{4} q^{4}+A_{6} q^{6}+\ldots}
$$

In the elastic region one may use $\delta_{\Gamma} \equiv \delta_{0}^{0}$ and then substitution of $\delta_{0}^{0}$ from (4) into (2) leads to integrals which are suitable for an explicit calculation in the framework of the theory of residua (for details see [5]). Finally the explicit form of the pion scalar FF is:

$$
\Gamma_{\pi}(t)=P_{n}(t) \frac{\left(q-q_{1}\right)}{\left(q+q_{2}\right)\left(q+q_{3}\right)\left(q+q_{4}\right)\left(q+q_{5}\right)} \times \frac{\left(i+q_{2}\right)\left(i+q_{3}\right)\left(i+q_{4}\right)\left(i+q_{5}\right)}{\left(i-q_{1}\right)}
$$

where $q_{i}$ are singularities of the FF to be placed in the upper or lower half q-plane and $P_{n}(t)$ is any polynomial normalized at $t=0$ to one.

\section{Results}

Results of the fit to the output $S$-wave amplitude from [3] are presented on Fig. 1. Corresponding parameters $A_{i}$ and positions of the poles and zeros are presented in Table 1 . The parameter $A_{1}$ has been fixed to be equal to the $\pi \pi$ scalar-isoscalar scattering length $a_{0}^{0}=0.220$ found in [2] and [4]. As is seen from Table 1 only 5 parameters (in fact 4 free) were enough to describe the elastic region and in result only 2 complex poles and one zero in the complex momentum plane are enough to construct full mathematical structure of the FF in the elastic region. They are presented on Fig 2.

On Fig. 1 presented is also behavior of modulus of the pion scalar form factor in the region $-3 \mathrm{GeV}^{2}<t<3 \mathrm{GeV}^{2}$. Physically may be interpreted region only below $990 \mathrm{MeV}\left(t \approx 0.98 \mathrm{GeV}^{2}\right)$

Noteworthy is that value of the squared radius of the presented FF is $r^{2}=0.765_{-0.087}^{+0.100} \mathrm{fm}^{2}$ what exceeds representative value $0.61 \pm 0.04 \mathrm{fm}^{2}$ presented in [8] and obtained on the basis of dispersion 

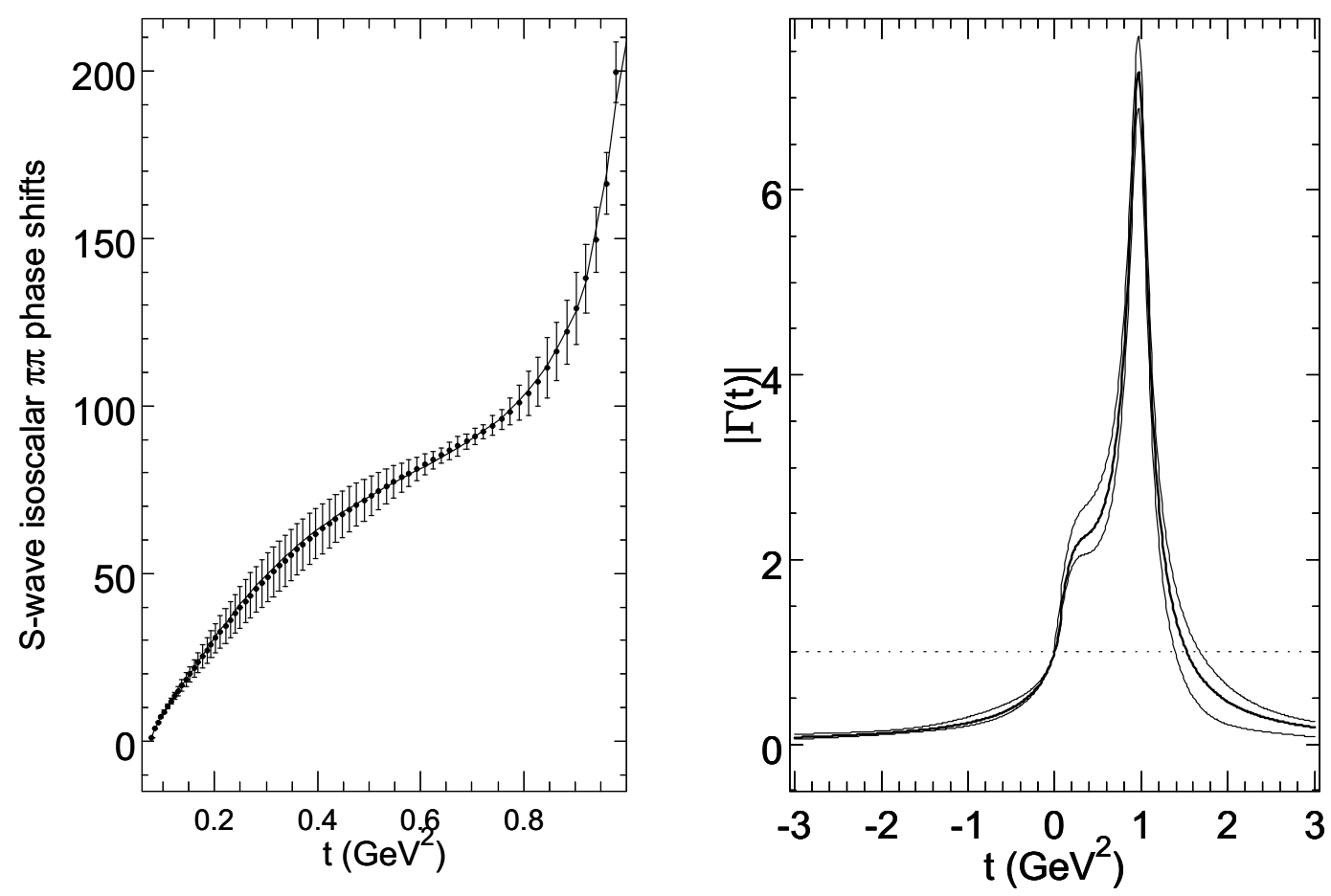

Figure 1. Left figure: the data on output $\delta_{0}^{0}(t)$ from [3] with theoretical errors to be generated by parameterization of the GKPY equations for the $S 0$ wave in [3]. Solid line represents performed in the presented analysis fit. Right figure: behavior of the pion scalar form factor in the region $-3 \mathrm{GeV}^{2}<t<3 \mathrm{GeV}^{2}$. Lines represent central values and upper and lower limits. Physically may be interpreted region only below $t \approx 0.98 \mathrm{GeV}^{2}$.

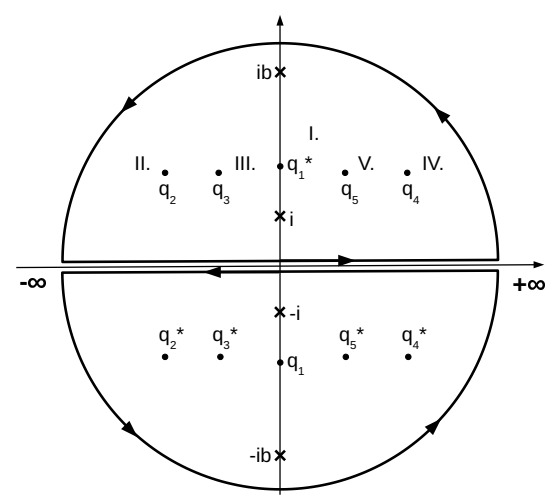

Figure 2. Schematic positions of poles $(\times)$ and branch points $(\bullet)$ of the $\Gamma_{\pi}(t)$ with contours of integrations in the upper and the lower half $q$-planes. 
Table 1. Left table: results from fit to the output points phase shifts from [3]. Positions of poles are in MeV.

Right table: positions of singularities of the pion-scalar FF in pion mass.

\begin{tabular}{c|c}
\hline$A_{1}$ & 0.220 fixed \\
$A_{3}$ & $0.151 \pm 0.020$ \\
$A_{5}$ & $-0.0144 \pm 0.0016$ \\
$A_{2}$ & $-0.055 \pm 0.038$ \\
$A_{4}$ & $-0.0089 \pm 0.0048$ \\
$f_{0}(500)$ & $451 \pm 14-i 260 \pm 33$ \\
$f_{0}(980)$ & $988 \pm 81-i 52 \pm 32$ \\
\hline
\end{tabular}

\begin{tabular}{c|r|c} 
& \multicolumn{1}{|c|}{$\operatorname{Re} q_{i}$} & \multicolumn{1}{|c}{$\operatorname{Im} q_{i}$} \\
\hline$q_{1}$ & \multicolumn{1}{|c}{0.00} & $-1.943 \pm 0.20$ \\
$q_{2}$ & $3.397 \pm 0.40$ & $0.196 \pm 0.04$ \\
$q_{3}$ & $-3.397 \pm 0.40$ & $0.196 \pm 0.04$ \\
$q_{4}$ & $1.385 \pm 0.10$ & $1.085 \pm 0.12$ \\
$q_{5}$ & $-1.385 \pm 0.10$ & $1.085 \pm 0.12$
\end{tabular}

theory. The value of the $r^{2}$ in presented here pion-scalar FF can be, however, significantly modified by for example adding two more parameters $A_{6,7}$ which lead to new pair of conjugated zeroes $q_{6}$ and $q_{7}$ or by simply multiplying FF by phase factor (nonzero only above the $K \bar{K}$ threshold) which leads to the phase of the $\Gamma_{\pi}(t)$ above the $K \bar{K}$ threshold very similar to that presented in [9] (see fig. 1 therein). Detailed analysis of these two methods will be presented in forthcoming publication.

In result first full and very simple construction of the pion scalar-isoscalar form factor has been presented. Only two poles (plus two conjugated ones) and one zero were needed to sufficiently precisely determine this form factor. Detailed analysis of the phase shifts used in performed fits (taken from dispersive analysis of the experimental data in the elastic $\pi \pi$ region) led to quite precise determination of positions of the $f_{0}(500)$ and $f_{0}(980)$ resonances presented in Table 1 .

Interesting and noteworthy is correct energy dependence of the form factor just below the $K \bar{K}$ threshold where presented analysis ends. This greatly enhances the accuracy and reliability of the presented parameterization of the form factor.

\section{Acknowledgements}

The support of the Slovak Grant Agency for Sciences VEGA under Grant No. 2/0158/13 and of the Slovak Research and Development Agency under the contract No. APVV-0463-12 is acknowledged. This work has been also partially supported by the Polish Science Center (NCN) Grant No. Dec-2013/09/B/ST2/04382.

\section{References}

[1] G. Colangelo, J. Gasser, and H. Leutwyler, Nucl. Phys. B 603, 125 (2001); B. Ananthanarayan, G. Colangelo, J. Gasser, and H. Leutwyler, Phys. Rep. 353, 207 (2001)

[2] I. Caprini, G. Colangelo, H. Leutwyler, Phys. Rev. Lett. 96, 132001 (2006)

[3] R. Garcia-Martin, R. Kamiński, J. R. Pela'ez, J. Ruiz de Elvira and F. J. Yndurain, Phys. Rev. D 83, 074004 (2011)

[4] R. Garcia-Martin, R. Kamiński, J. R. Pela'ez and J. Ruiz de Elvira, Phys. Rev. Lett. 107, 072001 (2011)

[5] S. Dubnicka, A. Z. Dubnickova, A. Liptaj, Phys. Rev. D 90, 114003 (2014)

[6] K. A. Olive et al (Part. Data Group) Chin. Phys. C 38, 090001 (2014)

[7] J. Gasser and U-G. Meissner, Nucl. Phys. B 357, 90 (1991)

[8] J. F. Donoghue, J. Gasser, H. Leutwyler, Nucl. Phys. B 343, 341 (1990)

[9] B. Ananthanarayan, I. Caprini, G. Colangelo, J. Gasser, and H. Leutwyler, Phys. Lett. B 602, 218 (2004) 